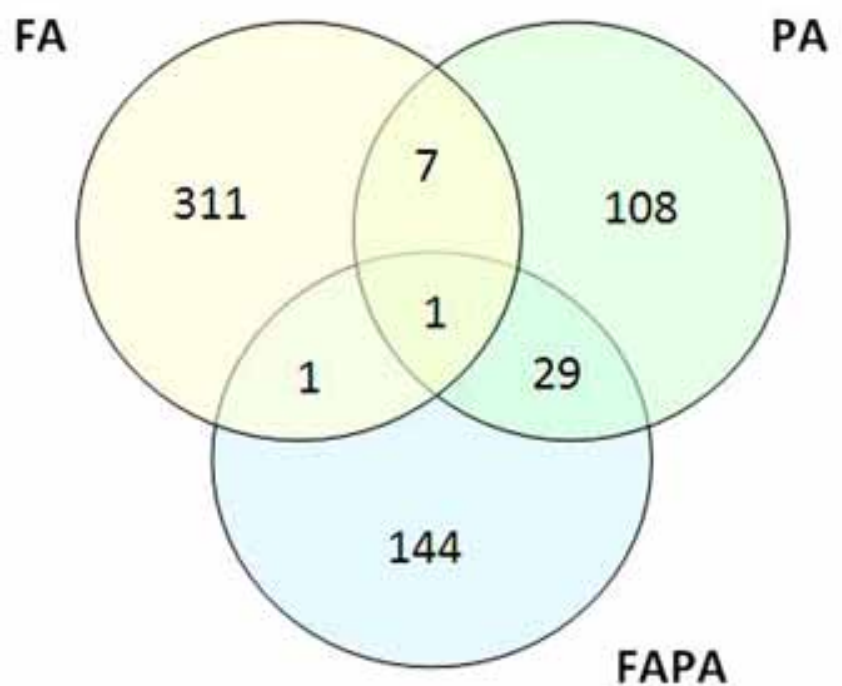

Abstract 730 Figure 2 Number of differentially expressed transcripts compared to control $(p<0.01)$, $96 \mathrm{~h}$ after birth

Many of these transcripts are involved in synaptic transmission and metabolism. Interestingly, we also found changes in several histone clusters and histone deacetylases.

Conclusions This is the first study to investigate whole genome expression in a preconditioning and ashyxia model that includes the fetal-to-neonatal transition and therefore truly resembles perinatal asphyxia. Our results warrant further research into epigenetic mechanisms of neuroprotection.

\section{PRADER WILLI SYNDROME (PWS) - PARTICULAR MOLECULAR PROFILE AND DIAGNOSTIC PROTOCOL IN ROMANIA}

doi:10.1136/archdischild-2012-302724.0731

${ }^{1} \mathrm{IE}$ Jurca- Simina, ${ }^{1} \mathrm{M}$ Gafencu, ${ }^{2} \mathrm{D}$ Dan, ${ }^{3} \mathrm{M}$ Puiu. ${ }^{1}$ Paediatrics, University of Medicine and Pharmacy, Timisoara; ${ }^{2}$ President of Romanian Prader Willi Association, Zalau; ${ }^{3} G e n e t i c s$, University of Medicine and Pharmacy, Timisoara, Romania

Background and Aims PWS-a rare genetic disease with a 1/12000-1/15000 newborns frequency, caused by deletion of some genes on paternal origin or maternal disomy of $15^{\prime}$ th chromosome. Main clinical manifestations are: neonatal hypotonia, excessive childhood feeding, obesity, cognitive deficiencies. Our aims are to implement molecular genetic/epigenetic tests and to develop interdisciplinary clinical research protocol, which allow early diagnostic and intervention in Romania.

Materials and Methods This study is part of a multicenter research project (CNMP/Partnerships, 2008-2011), on 19 Romanian PWS patients, 12 females, 7 males, between 6 months and 29 years. For diagnostic, were used major and minor criteria (Gunay-Aygun) as clinical methods and 5 genetic tests.

Results All patients have a clinical diagnostic score above 5, 63\% of them having a maximal major criteria number with $100 \%$ neonatal hypotonia, 95\% feeding difficulties at infants and hyperphagia after and a BMI till $60.2 \mathrm{~kg} / \mathrm{cm}^{2} .15 \%$ of patients have all minor criteria positive, with lethargy at infants, viscous saliva and small extremities predominance. $5 \%$ of patients have a positive $15 q 11-q 13$ microdeletion, $79 \%$ a FISH positive and for $47 \%$ patients MS-PCR is positive. Techniques like MS-MLPA were late introduced in Romania, $20 \%$ of our patients having them.

Conclusions The study indicates a relative correlation between clinical score and cytogenetic/molecular PWS confirmation and emphasizes the importance of early diagnostic. Interdisciplinary clinical criteria, karyotype, FISH and methylation analysis (MSPCR, MS-MLPA) are the main steps for a successful diagnostic protocol. Genetic tests results show a particular molecular profile in Romania with only $47 \%$ positive methylation results unlike literature (99\%).

\section{TURNER SYNDROME: A CLINICO-CYTOGENETIC STUDY OF 37 CHILDREN}

doi:10.1136/archdischild-2012-302724.0732

${ }^{1} \mathrm{M}$ Kammoun, ${ }^{1} \mathrm{~S}$ Mougou, ${ }^{2} \mathrm{R}$ Brahem, ${ }^{3} \mathrm{~N}$ Ghali, 'I Bel Haj Hmida, 'S Dimassi, ${ }^{4} \mathrm{~N}$ Soyah, ${ }^{1} \mathrm{H}$ El Ghzel, 'A Saad. 'Departments of Cytogenetics and Reproductive Biology, Farhat Hached University Teaching Hospital; '2Departments of Endocrinology, Farhat Hached University Teaching Hospital; ' ${ }^{3}$ Department of Nephrology, Sahloul University Teaching Hospital; " Departments of Pediatrics, Farhat Hached University Teaching Hospital, Sousse, Tunisia

Turner syndrome (TS) is defined by total or partial loss of the sex chromosomes X. Features vary widely including short stature and ovarian failure inconstantly associated with characteristic face, skeletal malformations, renal and cardiac anomalies and endocrine disorders.

We analyzed the clinical and cytogenetic profiles of 37 TS children diagnosed with TS from January 2007 to December 2011 in the aim to establish genotype- phenotype correlations.

Growth delay and hypothyroidism were noted respectively in $89.2 \%$ and $19.4 \%$ of patients. Diabetes and celiac disease was observed in $5.6 \%$ of cases. $35 \%$ of our cohort had a 45 , X karyotype, $8.1 \%$ had $45, \mathrm{X} / 47, \mathrm{XXX}$ mosaïcism and $5.4 \%$ have $45, \mathrm{X} / 46, \mathrm{XY}$ mosaïcism Interestingly, FISH revealed the presence of $S R Y$ gene. The remainders had structural abnormalities: $35.1 \%$ had isochromosome Xq which was homogenous in roughly half of cases. $10.8 \%$ were diagnosed with a terminal deletion Xp and $5.4 \%$ with a ring of chromosome X.

There was no correlation between genotypes and clinical features. The short stature in girls with TS is thought to be related to the haploinsufficiency of the SHOX gene on Xp22.3. As a result, treatment with GH is now routinely adopted even if the GH hormone is normally secreted. The higher risk of autoimmune diseases in women with TS could result from haploinsufficiency of the FOXP3 gene on Xp 11.23.

Otherwise, we highlight the importance of detection of $45, \mathrm{X} / 46$, Xy mosaïcism which may be cryptic requiring SRY probe FISH screening a condition that exposes to gonadoblastome and special chirurgical preventive treatment.

\section{BACTERIAL 16S RNA GENETIC MARKERS FOR FECAL SAMPLES TO DIFFERENTIATE CHOLEDOCHAL CYST FROM BILIARY ATRESIA}

doi:10.1136/archdischild-2012-302724.0733

T Okada, S Honda, H Miyagi, A Taketomi. Department of Gastroenterological Surgery I, Hokkaido University Graduate School of Medicine, Sapporo, Japan

Background and Aims Microbiota in fecal content from choledochal cyst (CC) and biliary atresia (BA) individuals at the operation were compared using $16 \mathrm{~S}$ rRNA gene libraries and terminal restriction fragment length polymorphism (T-RFLP).

Methods From 2002 to 2011, 1 infant with CC and 7 infant with BA (infants $\leq 2$ months of age) were treated at our institute. Fecal samples were obtained at the radical operation for CC and BA. Total fecal DNA was isolated and PCR was performed. The amplification of the fecal $16 \mathrm{~S}$ rDNA, restriction enzyme (BsII), size-fractionation of T-RFs and T-RFLP data analysis were performed. To compare the T-RFLP patterns among samples between CC and BA patients, the 
dissimilarity index which indicate the percentage of the peak area of the fragment (operational taxonomic unit: OTU) was used.

Results Two fecal samples of $1 \mathrm{CC}$ infant and 14 fecal samples of 7 BA infants were obtained. Nine predominant OTUs were detected with Bsl I digestion. The microbiota consisted of microbial communities of Bifidobacterium, Lactobacillales, Bacteroides, Prevotella, Clostridium clusters IV, XI, and XVIII, and Clostridium subcluster XIVa. The Bifidobacterium, Bacteroides, and Clostridium clusters were detected predominatly in CC than BA group. Lactobacillales was most predominat group in BA feces.

Conclusions Bacterial DNA showed marked differences in the composition of fecal microbiota in CC and BA infants. Molecular analysis of colonic microbiota using $16 \mathrm{~S}$ rRNA gene libraries and T-RFLP might be useful to differentiate CC from BA.

\section{ASSESSMENT OF DNA DAMAGE USING COMET ASSAY AND DETECTION OF OXIDATIVE STRESS PARAMETERS IN DOWN SYNDROME}

doi:10.1136/archdischild-2012-302724.0734

${ }^{1} \mathrm{G}$ El-Saeed, ${ }^{2} \mathrm{~S}$ Abdel Maksoud. ${ }^{1}$ Medical Biochemistry; ${ }^{2}$ Clinical Pathology, National Research Center, Giza, Egypt

Background Down syndrome is one of the commonest numerical chromosomal aberrations. Recent studies showed that oxidative stress is an important pathological factor in Down syndrome.

Objective To estimate the level of oxidative stress and DNA damage in Down syndrome patients.

Patients and methods: Fifteen Egyptian patients clinically diagnosed and cytogenetically proven to have Down syndrome. Fifteen Egyptian healthy controls were recruited from the outpatient clinic of Clinical Genetics Department, National Research Centre. Oxidative stress parameters including total antioxidant capacity (TAC), Superoxide dismutase (SOD) enzyme activity and Malondialdehyde (MDA) biomarkers were estimated. DNA damage was determined using the alkaline comet assay.

Results The MDA and SOD levels in Down syndrome patients were significantly higher than control group $(p=0.000 \& 0.01$, respectively). Total antioxidants levels were non-significantly higher than control group $(p=0.54)$. Statistical analysis of DNA damage levels in DS patients compared to controls showed significant increased levels $(p=0.000)$. There was a positive correlation of DNA damage levels with age in DS patients but not reaching a significant value $(\mathrm{p}=0.536)$. A non-significant positive correlation was detected between DNA damage levels and both MDA and TAC levels ( $p=0.8 \& 0.37$, respectively). Also a non-significant negative correlation of DNA damage levels with SOD levels was noticed $(\mathrm{p}=0.14)$.

Conclusion Oxidative stress plays a major role in DS pathogenesis.

\section{CONTRIBUTION OF ALKYLATING AGENTS IN THE CYTOGENETIC DIAGNOSIS OF FANCONI ANEMIA}

doi:10.1136/archdischild-2012-302724.0735

S Bouraoui, S Mougou, 0 Mamai, H Elghezal, A Saad. Department of Cytogenetic and Reproductive Biology, Farhat Hached University Teaching Hospital, Sousse, Tunisia

Background and Aims Fanconi Anemia (FA) is an autosomal recessive disease characterized by heterogenous phenotype which includes a bone marrow failure, diverse abnormalities and increased predisposition to develop leukemia. The cytogenetic diagnosis of FA cells to bifunctional alkylating agents, resulting in greatly increased chromosomal breakage and radial stuctures induced by cross-linking agents. To estimate the sensitivity and the specificity of the Mitomicyn C (MMC) and the Diepoxybutan (DEB), two alkylating agents used in the diagnosis of the FA, we studied the chromosomal instability on 22 patients using variable types and concentrations of these alkylating ( 25 and $40 \mathrm{ng} / \mathrm{ml}$ of MMC, $0.1 \mu \mathrm{g} / \mathrm{ml}$ of DEB).

Methods Heparanized venous blood samples were collected and were processed for the cytogenetic methodology in this study. After culture, 100 of metaphases were analysed to evaluate the frequency of chromosomal aberrations.

Results The MMC test at $25 \mathrm{ng} / \mathrm{ml}$ was High sensitive for FA. The DEB test showed a better specificity. The study of the mitotic segregation of sexual chromosomes by FISH took away any abnormality of the segregation to cells FA.

Conclusions A molecular study of the sensivity and the specificity of the alkylating agents used according to the group of complementation will come refine the diagnosis of FA by establishing a gold standard.

\section{CLINICAL AND CYTOGENETIC STUDY IN 116 CHILDREN COHORT WITH SEXUAL AMBIGUITY}

doi:10.1136/archdischild-2012-302724.0736

'I Ben Hadj Hmida, 'S Dimassi, 'M Kammoun, ${ }^{2} \mathrm{~K}$ Ben Helal, ${ }^{1} \mathrm{H}$ Hannechi, ${ }^{3} \mathrm{H}$ Seboui, 'M Gribaa, 'S Mougou-Zerelli, 'A Saad. 'Departments of Cytogenetics and Reproductive Biology, Farhat Hached University Teaching Hospital; 2 Departments of Pediatic, Hospital Ibn El Jazar Kairouan; ${ }^{3}$ Departments of Neonatology, Farhat Hached University Teaching Hospital, Sousse, Tunisia

Disorder of sex development may result from multiple etiologies, led to many physical impacts involving genital abnormalities. Differentiation of a testis or an ovary from a bipotential gonad is a complex developmental process where various genes and hormones interact. Thus, the causality of sexual ambiguity was supported by the presence of many chromosomal abnormalities, especially when sex determination genes were involved.

A careful clinical examination will primarily interest to define the sexual ambiguity Prader score and the whole genital and extra genital malformation context.

Our study focuses on cases referred to the department of Cytogenetics and Reproductive Biology CHU Farhat Hached for ambiguous genitalia cytogenetic exploration from January 2010 to February 2012.

The 116 patients studied with ambiguous genitalia of varying severity, $80 \%$ were younger than 10 years. $7.5 \%$ of them showed chromosomal abnormalities.

In 3 patients, the chromosomal abnormality involved the autosomes 4, 5 and 1 with a $4 p$ deletion in Wolf-Hirschhorn syndrome, deletion $5 p$ in Cri-du-chat syndrome and $1 q$ deletion revealed by Comparative Genomic Hybridization-array. Theses anomalies are associated with loss of genital differentiation protein function. In 4 other patients abnormal sexual chromosome number was identified. For 86 children with normal karyotypes 46, XY, SRY gene molecular cytogenetic screening was performed. A deletion may in these cases explain the phenotype.

Identification of gene rearrangements causing urogenital birth defects can have an impact on the diagnosis and genetic counseling. Thus more study is necessary to find new candidate genes in signaling pathways regulating sexual differentiation.

\section{MOLECULAR CYTOGENETIC AND COMPARATIVE GENOMIC HYBRIDIZATION ANALYSIS IN NEWBORNS WITH CONGENITAL HEART DISEASES}

doi:10.1136/archdischild-2012-302724.0737

'I Malek, 'H Ben Khelifa, "I Ben Abdallah, 'H Hannen, 'S Mougou-Zerelli, ${ }^{2} \mathrm{H}$ Seboui, ${ }^{1} \mathrm{H}$ El Ghezal, 'A Saad. 'Departments of Cytogenetics and Reproductive Biology, Farhat Hached University Teaching Hospital; ' $N$ Neonatology Service, Farhat Hached University Teaching Hospital Sousse, Sousse, Tunisia

Congenital heart diseases (CHD) are abnormalities that occur at birth and resulting from a developmental defect during cardiac 